Dinkler, L., Lundström, S., Gajwani, R., Lichtenstein, P., Gillberg, C. and Minnis, H. (2017) Maltreatment-associated neurodevelopmental disorders: a co-twin control analysis. Journal of Child Psychology and Psychiatry, 58(6), pp. 691-701. (doi:10.1111/jcpp.12682)

There may be differences between this version and the published version. You are advised to consult the publisher's version if you wish to cite from it.

This is the peer-reviewed version of the following article: Dinkler, L., Lundström, S., Gajwani, R., Lichtenstein, P., Gillberg, C. and Minnis, H. (2017) Maltreatment-associated neurodevelopmental disorders: a cotwin control analysis. Journal of Child Psychology and Psychiatry, 58(6), pp. 691-701, which has been published in final form at 10.1111/jcpp.12682. This article may be used for non-commercial purposes in accordance with Wiley Terms and Conditions for SelfArchiving.

http://eprints.gla.ac.uk/131295/

Deposited on: 17 November 2016

Enlighten - Research publications by members of the University of Glasgow http://eprints.gla.ac.uk 


\title{
Maltreatment-Associated Neurodevelopmental Disorders: A Co-Twin Control Analysis
}

\author{
Lisa Dinkler ${ }^{1}$ \\ Sebastian Lundström ${ }^{1,3}$ \\ Ruchika Gajwani $^{2}$ \\ Paul Lichtenstein ${ }^{4}$ \\ Christopher Gillberg ${ }^{1,2}$ \\ Helen Minnis ${ }^{1,2}$
}

\section{Author Affiliations:}

${ }^{1}$ Gillberg Neuropsychiatry Centre, University of Gothenburg, Gothenburg, Sweden

${ }^{2}$ Institute of Health and Wellbeing, University of Glasgow, Glasgow, UK

${ }^{3}$ Centre for Ethics, Law and Mental Health (CELAM), University of Gothenburg, Gothenburg,

Sweden

${ }^{4}$ Department of Medical Epidemiology and Biostatistics, Karolinska Institutet, Stockholm, Sweden

Word count: 7,537

Access to data: Lisa Dinkler had full access to all the data in the study and takes responsibility for the integrity of the data and the accuracy of the data analysis.

Conflict of interest: The authors declare no conflict of interest.

\section{RUNNING HEAD:}

Maltreatment-Associated Neurodevelopmental Disorders 


\section{Abstract}

Background: Childhood maltreatment (CM) is strongly associated with psychiatric disorders in childhood and adulthood. Previous findings suggest that the association between CM and psychiatric disorders is partly causal and partly due to familial confounding, but few studies have investigated the mechanisms behind the association between $\mathrm{CM}$ and neurodevelopmental disorders (NDDs). Our objective was to determine whether maltreated children have an elevated number of NDDs, and whether CM is a risk factor for an increased NDD "load" and increased NDD symptoms when controlling for familial effects.

Methods: We used a cross-sectional sample from a population-representative Swedish twin study, comprising 8,192 nine-year old twins born in Sweden between 1997 and 2005. CM was defined as parent-reported exposure to emotional abuse/neglect, physical neglect, physical abuse and/or sexual abuse. Four NDDs were measured with the Autism-Tics, AD/HD, and other Comorbidities inventory. Results: Maltreated children had a greater mean number of NDDs than non-maltreated children. In a co-twin control design, CM-discordant monozygotic twins did not differ significantly for their number of NDDs, suggesting that CM is not associated with an increased load of NDDs when genetic and shared environmental factors are taken into account. However, CM was associated with a small increase in symptoms of Attention-Deficit/Hyperactivity Disorder and Autism Spectrum Disorder in CM-discordant monozygotic twins, although most of the covariance of CM with NDD symptoms was explained by common genetic effects.

Conclusions: Maltreated children are at higher risk of having multiple NDDs. Our findings are, however, not consistent with the notion that CM causes the increased NDD load in maltreated children. Maltreated children should receive a full neurodevelopmental assessment and clinicians should be aware that children with multiple NDDs are at higher risk of maltreatment.

Keywords: Child maltreatment, child abuse, neurodevelopmental disorders, behaviour genetics, cotwin control design

Abbreviations: Childhood maltreatment (CM), Neurodevelopmental Disorders (NDDs) 
A large body of research has linked Child Maltreatment (CM), including physical, emotional, and sexual abuse, physical and emotional neglect, with a broad range of psychiatric disorders in childhood and adulthood (Jones et al., 2012; Kessler et al., 2010; Norman et al., 2012). CM occurring together with neurodevelopmental disorders (NDDs) has been proposed, in clinical research, to be associated with a greater than expected number of NDDs (Minnis, 2013). This notion has been supported by a small study showing elevated numbers of NDDs in severely maltreated UK adoptees (Kocovska et al., 2012), but has not yet been confirmed in a population-representative sample that would include lesssevere CM. During the last decades it has been increasingly recognized that NDDs share symptomatology and aetiology with each other and that one diagnosis in childhood may be labelled as another disorder in adulthood (Gillberg, 2010). The association between CM and psychopathology furthermore seems to reflect a general vulnerability for any psychiatric disorder rather than for a specific disorder (Kessler et al., 2010). These aspects highlight the need for broader assessments of NDDs and other psychiatric problems when studying CM and its links with psychiatric disorder. In addition, the Adverse Childhood Experiences (ACE) studies have revealed the usefulness of examining the cumulative influence of early childhood adversities (Felitti et al., 1998), but this thinking has not previously been applied to the examining the "load" of early childhood NDDs.

Secondly, it has not yet been well established whether CM indeed is an environmental factor with causal effects on psychiatric disorders, or on specific groups of disorders. The results of association studies often tend to be interpreted as if $\mathrm{CM}$ were a causal environmental factor, however any relationship between risk factors and outcomes may be confounded by familial factors (genetic, shared environmental, or both) that are correlated to both exposure and outcome, also implying that there is genetic influence on supposedly "purely” environmental measures (D'Onofrio et al., 2013).

Whether a factor is causal or not has to be tested using family-based, quasi-experimental study designs. If the association between two factors holds after controlling for familial confounding, a true environmental risk association is implied, although temporal sequencing also has to be ascertained in longitudinal designs to verify causality (D'Onofrio et al., 2013). Indeed, previous child-based twin studies (i.e. the genes of children of varying degrees of genetic relatedness are the unit of 
measurement; Neiderhiser et al., 2004) have suggested that the child's experience of parent behaviours and of stressful live events including CM is partly heritable (Jaffee, Caspi, Moffitt, Polo-Tomas, et al., 2004; Kendler \& Baker, 2007). Since child-based designs measure the role of genetic factors in the elicitation of parental behaviour (Kendler \& Baker, 2007), the heritability estimates in CM might reflect an evocative gene-environment correlation (also: genetically mediated child effects; Narusyte et al., 2008; Schulz-Heik et al., 2010), where the genetically influenced behaviour of the child evokes a certain response in the parent.

Genetically sensitive studies on the effects of childhood traumatic experience on child and adult psychopathology have revealed mixed findings regarding a causal role of traumatic events. Co-twin control studies have indicated direct effects of childhood trauma, including sexual abuse, on e.g. depression, anxiety, substance abuse and eating disorders in adulthood (Brown et al., 2014; Kendler et al., 2000), but not on most of the personality disorders (Berenz et al., 2013). The well-known association between CM and adult criminality was almost completely accounted for by familial confounding when utilizing a co-twin control design (Forsman \& Långström, 2012). With regard to child psychopathology, risk effects of bullying on internalizing problems (Arseneault et al., 2008), and risk effects of $\mathrm{CM}$ and negative parenting on externalizing and antisocial behaviour have been indicated in genetically sensitive designs (Jaffee, Caspi, Moffitt, \& Taylor, 2004; Jonson-Reid et al., 2010; O'Connor et al., 1998). Other genetically sensitive studies have suggested conflicting findings that the association of parent-child hostility with Attention-Deficit/Hyperactivity Disorder (ADHD) symptoms and the association of CM with conduct problems were mainly due to common genetic factors (Lifford et al., 2009; Schulz-Heik et al., 2010).

Most studies examining a causal relationship between CM and child psychiatric outcomes have focussed on internalizing and externalizing problems, with a specific focus on the disruptive behavioural disorders - ADHD, oppositional-defiant disorder and Conduct Disorder (Jaffee, Caspi, Moffitt, \& Taylor, 2004; Jonson-Reid et al., 2010; Lifford et al., 2009). The English \& Romanian Adoptees Study did examine a broader range of outcomes including neurodevelopmental problems, but investigated severe early global deprivation (Kumsta et al., 2015). No study has investigated a 
possible risk effect of less-severe and more common CM in a population-representative sample focusing on a broader range of NDDs, including ADHD, Autism Spectrum Disorder (ASD), Learning Disorders, and Tic Disorders.

The current study asks three questions in a population-representative twin sample: (1) Do children exposed to CM have an increased NDD load compared to children unexposed to CM?; if so, (2) Is CM a risk factor for an increased NDD load when controlling for familial effects?; (3) Is CM a risk factor for increased symptoms of specific NDDs when controlling for familial effects? 


\section{Methods}

\section{Participants}

The present study is based on data from the Child and Adolescent Twin Study in Sweden (CATSS), an ongoing longitudinal study aiming to include all twins born in Sweden since July 1, 1992. Since 2004, the parents of all nine-year-old twins have been invited to participate in a telephone interview. The CATSS has been described in detail elsewhere (Anckarsater et al., 2011). Briefly, individuals born between $1^{\text {st }}$ July 1998 and $31^{\text {st }}$ December 2005 were included in the present study. Zygosity was ascertained by a panel of 48 single nucleotide polymorphisms. If DNA samples were not accessible, an algorithm of five questions on the similarity of the twins was applied. Cases with less than $95 \%$ probability of correct classification were not assigned any zygosity. In the present sample $26.7 \%$ $(3,578)$ of the twins were monozygotic (MZ), 34.5\% (4,614) dizygotic same-sex (DZ-ss), and 36.3\% $(4,860)$ dizygotic opposite-sex. For 330 individual cases $(2.5 \%)$ zygosity could not be determined. Individuals with unknown zygosity as well as dizygotic opposite-sex twins were excluded from all analyses, yielding a final study sample of $N=8,192$ twins $(50.6 \%[4,148]$ males). The CATSS has ethical approval from the Regional Ethical Review Board in Stockholm (Dnr 2010/597-31/1).

\section{Measures}

Childhood Maltreatment. CM was assessed with the Life Stressor Checklist-Revised (LSC-R; Wolfe \& Kimerling, 1997). The LSC-R is a 30-item self-report screening measure of potentially traumatic life events according to DSM-IV criteria for Posttraumatic Stress Disorder, and of other seriously stressful life events. For this study we used the five yes/no questions on the experience of CM from the LSC-R as reported by the parents. Emotional abuse/neglect, physical neglect, and physical abuse were assessed with one question each, and sexual abuse with two questions (see Appendix). If a CM event was endorsed in the LSC-R, follow-up questions were asked regarding the child's age at the event and the perpetrator. If one or more types of $\mathrm{CM}$ were indicated by the parent, the child was considered as having suffered CM. In the current study, the LSC-R is parent-reported, which might have introduced bias due to under- or overreporting. Sensitivity analyses and an extensive discussion 
regarding the validity of the $\mathrm{CM}$ measure applied in this study including reliability and validity data from previous studies can be found in the supplemental material.

Neurodevelopmental Disorders. All NDDs reported in this study were assessed with the Autism-Tics, $\mathrm{AD} / \mathrm{HD}$, and other Comorbidities inventory (A-TAC). The A-TAC is a fully structured 96-item parent-report telephone interview designed for large scale epidemiological purposes to screen for neurodevelopmental and other psychiatric disorders in childhood based on symptom criteria and wellknown clinical features. Items are scored as 1 (yes), 0.5 (yes, to some extent) and 0 (no) and are presented in a life-time perspective. The A-TAC has been validated against clinical diagnoses crosssectionally and longitudinally (Larson et al., 2010; Larson et al., 2013). The current study included four NDDs measured continuously (symptom loads). The internal consistencies (Cronbach's alpha) in the current sample were good to excellent for $\operatorname{ADHD}(.92,19$ items) and $\operatorname{ASD}(.83,17$ items), acceptable for Learning Disorders (.74, 3 items), and modest for Tic Disorders (.58, 3 items). The measurements of these NDDs were furthermore employed in a categorical fashion in order to generate two further outcome variables: 1) number of NDDs (broad definition) applying screening cut-offs (with higher sensitivity than specificity) based on previous research: ADHD ( $\geq 6.0$, Sensitivity 0.98/Specificity 0.81), ASD ( $\geq 4.5,0.96 / 0.88)$, and Learning Disorders ( $\geq 1.0,0.88 / 0.75)$; and 2$)$ number of NDDs (narrow definition), applying cut-offs validated as proxies to clinical diagnoses (with higher specificity than sensitivity): $\operatorname{ADHD}(\geq 12.5$, Sensitivity $0.52 /$ Specificity 0.95$)$, ASD $(\geq 8.5$, 0.71/0.95), and Learning Disorders ( $\geq 3.0,0.23 / 0.96$; Larson et al., 2010). For Tic Disorders only one cut-off has been validated $(\geq 1.5,0.92 / 0.90)$, which therefore has been used for both summary variables. The outcome number of NDDs in the text generally refers to number of NDDs (broad definition), except where explicitly stated otherwise.

\section{Data Analyses}

To investigate if maltreated children have increased NDD load, we compared the number of NDDs, as well as the symptom loads of ADHD, ASD, Learning Disorders and Tic Disorders between individuals exposed (CM) and unexposed to $\mathrm{CM}$ (non-CM), applying independent samples t-Tests. 
To assess if CM is a risk factor for an increased NDD load when controlling for familial effects, we applied a co-twin control design. The co-twin control method is designed to investigate causal inferences in observational research, in other words, if there is a true causation (environmental factor) or familial confounding (McGue et al., 2010). Co-twins of twin pairs differentially exposed to CM (discordant) are compared for differences in their number of NDDs (within-pair effects). MZ twins are $100 \%$ genetically identical, while DZ twins on average share $50 \%$ of their segregating alleles.

Furthermore, twin pairs are matched for common environmental influences (shared environment) since they are expected to be reared under the same conditions of a family (e.g. parental socioeconomic status, parental education level). Thus, the effect of CM on the number of NDDs can be estimated while accounting for unmeasured confounding factors, i.e., all genetic and environmental factors shared within twin pairs.

To estimate these within-pair effects, conditional linear regression with cluster-robust standard errors was applied, where each twin pair is treated as a cluster and the number of NDDs is regressed on CM within each cluster (Neuhaus \& McCulloch, 2006). The effects within discordant MZ pairs and DZ-ss pairs are then compared to the association of CM and the number of NDDs in the population, which was estimated with linear regression (number of NDDs in all maltreated children versus number of NDDs in all non-maltreated children taking no account of twin pair relatedness or zygosity).

If the association of CM with number of NDDs was entirely causal, we would expect the effects to be of similar size on the population level and within discordant MZ pairs and DZ-ss pairs, as genetic similarity and shared environmental factors do not have any influence. If, however, the within-pair effects are attenuated compared to the effect on the population level, familial confounding is implied. The comparison between discordant MZ twins is the most powerful one, because it completely controls for genetic and shared environmental effects. If there is no difference at all in number of NDDs within $\mathrm{MZ}$ twin pairs discordant for $\mathrm{CM}$, the association between $\mathrm{CM}$ and number of NDDs in the population would be entirely due to familial confounding. A partly causal effect and partly familial confounding of the relationship between CM and number of NDDs would be suggested if the withinpair effect in discordant MZ pairs is lower than in discordant DZ-ss pairs, but still different from zero. 
If the size of the within-pair effects is similar in discordant MZ and DZ-ss pairs, but lower than the effect on the population level, the association is confounded by shared environmental effects (McGue et al., 2010).

To answer the third research question we conducted the co-twin control analyses separately for the symptom load of each NDD. We also illustrated the number of NDDs in maltreated versus nonmaltreated twins in a graphic fashion. IBM SPSS Statistics and OpenMx were used for all analyses; a significance level of $\alpha<.05$ was applied throughout. 


\section{Results}

In total, 374 individuals were reported as having been maltreated, yielding a population prevalence of $4.6 \%$, and a male-female ratio of 1.7:1 for $\mathrm{CM}$ as defined in the present context. The occurrence of the specific CM types was unevenly distributed (emotional abuse/neglect $n=286$, physical neglect $n=48$, physical abuse $n=109$, sexual abuse $n=23$ ), with substantial overlap across categories. The prevalence of CM did not differ across zygosities (MZ 4.3\% [155], DZ-ss 4.7\% [219], $p=0.3934$ ). The tetrachoric correlations (correlation of $\mathrm{CM}$ in twin 1 with $\mathrm{CM}$ in twin 2) were quite similar for MZ and DZ-ss twin pairs (MZ: 0.93, 95\% CI 0.90-0.97, DZ-ss: 0.86 95\% CI .80-.91), which was mirrored in the univariate liability threshold analyses where the largest variance component was the shared environment $\left(c^{2}=0.77,95 \%\right.$ CI $\left.0.66-0.88\right)$, followed by the heritability estimate $\left(h^{2}=0.15\right.$, 95\% CI 0.02-0.29).

Fifty-eight percent of the maltreated individuals experienced CM within the nuclear family (biological, step or adoptive parents, or siblings were indicated as perpetrators), $36 \%$ had been maltreated by persons outside the nuclear family (other relatives or known children or adults), and $5 \%$ experienced both. The mean onset age for $\mathrm{CM}$ was 4.70 years (emotional abuse/neglect: 4.46, physical neglect: 3.29 , physical abuse: 5.21, and sexual abuse: 6.04), while the average parent-reported onset of any of the four NDDs was slightly higher at 5.06 years (ASD: 4.75, ADHD: 5.20, Learning Disorders: 6.38, Tic Disorders: 5.73).

The population prevalence of NDDs defined as the presence of at least one out of four NDDs (narrow definition), was $6.3 \%(n=518)$. Prevalence data for each NDD separately is provided in the supplement. Since males exhibited higher levels of both CM $(p<0.0001)$ and NDDs (Table 1) than females, the main analyses were stratified by sex.

\section{-Insert Table 1 about here-}


Research Question 1: Do children exposed to CM have an increased NDD load compared to children unexposed to $C M$ ?

On average, maltreated individuals had a higher number of NDDs than non-maltreated individuals. In the CM group, $24.1 \%$ had at least one NDD (narrow definition, applies throughout the paragraph), compared to $5.5 \%$ in the non-CM group $(p<.0001, \varphi=.16)$ (Table 1). The relative risk (RR) of being affected by two or more NDDs was seven times higher for maltreated individuals (8.3\%) than for nonmaltreated individuals $(1.2 \%$; $\mathrm{RR}=7.11 ; 95 \% \mathrm{CI}$ : 4.80-10.55). The relative risk of being affected by three or four NDDs was almost six times as high (2.1\% of CM vs. $0.4 \%$ of non-CM, RR $=5.76 ; 95 \%$ CI: 2.65-12.51). This was mirrored in the continuous analyses where maltreated males and females had significantly more symptoms of all NDDs compared to non-maltreated individuals.

\section{Research Question 2: Is CM a risk factor for an increased NDD load when controlling for familial} effects?

The number of NDDs of the maltreated MZ and DZ-ss twins was plotted against those of their cotwins in Figure 1. MZ twins differentially exposed to CM did not differ in the number of NDDs $(p=.199)$, while DZ-ss twins differentially exposed to CM did differ significantly in the number of NDDs $(p<.0001)$. The same pattern emerged when analysed separately for both male and female twins (male MZ: $p=.2800$, male DZ-ss: $p=.0002$; female MZ: $p=.4740$, female DZ-ss: $D=p=.0007)$. This indicates that the association between CM and the number of NDDs is almost completely explained by familial factors.

Research Question 3: Is CM a risk factor for increased symptoms of specific NDDs when controlling for familial effects?

Compared to their non-maltreated co-twins, maltreated male MZ twins had significantly more symptoms of ADHD (Difference in the unstandardized mean score on the A-TAC scale $[D]=1.47$; $p=.0372)$ and $\operatorname{ASD}(D=0.90 ; p=.0185$; Table $2 \&$ Figure 2$)$, suggesting a small risk effect of CM. Maltreated female MZ twins showed significantly more symptoms of $\operatorname{ASD}(D=0.67 ; p=.0044)$, and also tended to have more symptoms of Learning Disorders $(D=0.24 ; p=.0737$; effect size similar to that in ASD) than their non-maltreated co-twins, suggesting a small risk effect of CM. However, the 
results also imply substantial familial confounding of the relationship between $\mathrm{CM}$ and the symptom load of these NDDs, since the within-pair effects in discordant MZ twins are strongly attenuated compared to the effects on the population level.

There was a significant negative difference for symptoms of Tic Disorders in female MZ twins, so that non-maltreated female MZ twins had more symptoms than their maltreated co-twins $(p=.0171)$. The within-pair difference in discordant MZ twin pairs was near zero/non-significant for Learning Disorder and Tic Disorder symptoms in males and ADHD symptoms in females, suggesting complete confounding of the relationship of CM and these symptom loads by familial factors.

-Insert Table 2, Figure 1 and Figure 2 about here- 


\section{Discussion}

In a population-based sample of nine-year-old twins we found a significantly higher number of NDDs in maltreated individuals compared to non-maltreated individuals, in line with suggestions from previous clinical research (Kocovska et al., 2012). Utilizing the genetically informative co-twin control design, CM was not associated with an increased load of NDDs when genetic and shared environmental factors are taken into account. However, in the co-twin control analysis CM was associated with a small increase in symptoms of ADHD and ASD, although most of the covariance of CM with NDD symptoms seems to be explained by common familial factors. This apparently paradoxical finding might imply that the increase in ADHD and ASD symptomatology among maltreated co-twins is not substantial enough to be reflected in an increased NDD load, when NDDs are considered categorically as diagnoses - or that CM increases symptoms of existing diagnoses rather than leading to new diagnoses.

Due to the highly overlapping confidence intervals between effects on the population and within-pair levels, it was not possible to confidently distinguish genetic confounding and shared environmental confounding. On a univariate level, the shared environmental influence on CM was very high (.77), whereas shared environment has been shown to have little or no influence on NDDs (Anckarsater et al., 2011). This implies that shared environmental influences on the association between CM and NDD symptoms can statistically not arise. We therefore conclude that the co-occurrence of CM with a higher load of NDD symptoms seems to a large extent to be accounted for by a shared genetic liability, increasing both the risk of being maltreated and of having more co-occurring NDDs. This is in line with previous research showing that common genetic factors largely account for the covariation of parent-child hostility and child ADHD symptoms, corporal punishment and child antisocial behaviour, and of maltreatment and child conduct problems (Jaffee, Caspi, Moffitt, Polo-Tomas, et al., 2004; Lifford et al., 2009; Schulz-Heik et al., 2010). Our findings are furthermore supported by a recent study, applying comprehensive and rigorous testing across two longitudinal cohorts, which suggests that the association between childhood violence victimization and later cognitive deficits is 
"largely noncausal" (Danese et al., 2016). Future studies could estimate the magnitude of common genetic effects on the association of CM and NDDs applying traditional quantitative twin modelling.

Genetic confounding indicates that gene-environment correlation (commonly abbreviated rGE) is operating (Narusyte et al., 2008). rGE can be of different types, which can, however, not clearly be distinguished with a standard twin design. This can be accomplished using, for example, children-oftwins and adoption designs (Narusyte et al., 2008). In the observed association of CM with NDDs, passive rGE might play a role: the parents' genes related to their own NDD traits are correlated with their children's genetically influenced NDDs and might also increase the risk of the parents maltreating their child. Indeed it has been shown that parental liability to externalizing disorders is highly heritable and its intergenerational transmission accounts for most of the variance in childhood disruptive disorders (Bornovalova, Hicks, Iacono, \& McGue, 2010). Another explanation for the identified genetic confounding may be evocative rGE, in which genetically influenced traits of a child such as impulsivity or poor theory of mind could be evoking CM by the parent. Adoption studies have demonstrated evocative child effects for negative parenting, that is, an increasing likelihood of harsh, inconsistent and hostile parenting of adopted children with a high genetic risk for antisocial behaviour (O'Connor et al., 1998). Furthermore, as the present study also included extra-familial perpetrators of $\mathrm{CM}$, active rGE (where the child actively selects environments due to its genetically influenced characteristics) cannot be ruled out as influencing the association of CM with NDDs.

We found a very small increase in symptoms of ADHD and ASD in maltreated monozygotic twins as compared to their non-maltreated co-twins. This finding is in line with a recent study that found effects of CM on adult ADHD symptoms that were consistent with a causal interpretation (effect size 0.40 standard deviations in the population level and 0.18 standard deviations in discordant MZ twins; Capusan et al., 2016). There is growing evidence that CM contributes to structural and functional changes in the brain, specifically in the fronto-limbic regions, involved in emotion and motivation processing, as well as the fronto-striatal regions, mediating executive functioning such as attention, cognitive flexibility and behaviour regulation/inhibition (Hart \& Rubia, 2012; Teicher \& Samson, 2016). These changes might contribute towards some of the cognitive and behavioural difficulties 
found in NDDs (Hart \& Rubia, 2012). However, due to the lack of temporal resolution we were not able to examine the direction of a potential causal link between CM and ADHD/ASD symptoms.

We did not find indications for an effect of CM on ADHD symptoms for females. Indeed it has been reported that $\mathrm{CM}$ can have sex-specific effects in terms of morphological alterations of brain structures (for a review see Teicher \& Samson, 2016). For example, effects of stress exposure on hippocampal volume and effects of $\mathrm{CM}$ on reduced corpus callosal thickness (which also has been associated with ADHD; Luders et al., 2009) have been shown to be stronger in males than in females. Sex differences have also been shown previously in the genetic/environmental aetiology of child mental health problems, with males showing a much higher heritability of Attachment Disorder (Minnis et al., 2007). The counter-intuitive finding that CM was associated with significantly fewer symptoms of Tic Disorders in females when controlling for familial effects might be explained by children trying to suppress their tics when risking punishment for them by their parents. Alternatively, neglectful parents may not notice their child's tics and fail to report on them.

\section{Implications}

Irrespective of the underlying mechanisms, our results show that children who have experienced CM display more NDDs than children who have not experienced CM (cf. Kocovska et al., 2012).

Clinicians need to be aware of the increased risk for abuse or neglect in children with multiple NDDs. Our results also point in the direction that individuals presenting with apparently trauma-related disorders in child and adult psychiatry may have undiagnosed NDDs. Therefore a full diagnostic work-up for trauma-related disorder, in a patient of any age, must contain an assessment of NDDs including ADHD, ASD, Learning Disorders and Tic Disorders. It will therefore be essential to screen and assess for NDDs in any adult with psychiatric disorder where maltreatment has been present in childhood. The finding that the association between CM and NDDs was substantially explained by shared genetic factors has the very important clinical implication that many biological parents of children who are/have been maltreated may have untreated NDDs which should be properly treated. Although treating parents' neurodevelopmental needs will not spare children from developing NDDs 
themselves, it may at least spare them from also being maltreated. Given a possible role of evocative child effects on $\mathrm{CM}$, one needs to emphasize the importance of support for parents and of specific parenting training interventions for mothers and fathers of children with neurodevelopmental difficulties. In addition, the small effect of CM on elevated symptoms of ADHD and ASD (and possibly Learning Disorders) is clinically relevant, as it suggests that intervening to prevent CM may to some extent alleviate NDD-symptomatology and associated sequelae.

\section{Limitations and Strengths}

There are some limitations in the present study. Firstly, the psychometric properties of the A-TAC for measuring NDDs are good, but not perfect. Clinical assessments, however, are not feasible in largescale population studies such as the present one. Secondly, we have to suspect an underreporting of CM by the parents in our sample (see an extensive argumentation in the Supplement). Assigning falsenegative reported cases to the non-maltreated group could have biased our results in a conservative way. The ratio of parents of MZ twins who apparently underreported and parents of DZ twins who apparently underreported was, however, very similar to the ratio of MZ and DZ twins in the total sample, which minimizes the probability of a confounding by underreporting in the co-twin control design. Thirdly, non-response might have led to some bias, although the response rate was $70 \%$, and previous attrition analyses have demonstrated only small differences between responders and nonresponders (Anckarsater et al., 2011). Fourthly, we did not have sufficient information to investigate the temporal sequence of onset of symptoms of NDDs, therefore, we could not investigate the direction of causality in the association between CM and increased NDD symptoms that we found in monozygotic twins. It could be possible that CM leads to small increase in NDD symptoms, but it is also imaginable that more NDD symptoms will cause CM. Prospective studies would be needed to clarify the temporal sequence of the onset of CM and NDD symptoms and will require large general population datasets starting antenatally or in very early childhood which currently do not exist. The present study also has important strengths: the analyses benefited from the use of a well-validated instrument for the NDDs studied in a nationwide population-representative sample of twins with a high response rate. 


\section{Conclusions}

Many maltreated children carry a double burden, having both extremely adverse experiences in childhood and a high load of NDDs. Our data suggest that the association between CM and NDDs is mainly due to common genetic factors, suggesting that these might be the origin to both $\mathrm{CM}$ and NDDs. This is not consistent with the notion that CM increases NDD load in the maltreated children. An important clinical implication of our findings is that children who have been maltreated should always be investigated for NDDs. Similarly, when children have NDDs - particularly multiple NDDs

- clinicians should remember that such children are at higher risk of current or future maltreatment. It is therefore essential that parents of children with NDDs are able to access additional support. 


\section{Supporting information}

Additional supporting information may be found in the online version of this article.

1. Items applied from the Life Stressor Checklist-Revised to assess Childhood Maltreatment

2. Validity of the Childhood Maltreatment Measure

3. Table S1: Prevalence of Neurodevelopmental Disorders in \% [95\% CI] in 8,192 Swedish twins applying validated low and high cut-offs

4. Table S2: Effect of Childhood Maltreatment (CM) on symptoms of Neurodevelopmental Disorders (NDDs) in a Co-Twin Control Design (sex-collapsed)

\section{Acknowledgements}

The Child and Adolescent Twin Study in Sweden study is supported by the Swedish Research Council for Health, Working Life and Welfare, the Söderström-Königska Foundation, and the Swedish Research Council (Medicine and SIMSAM). The current study received no specific funding. The funding sources had no involvement in the study design, collection, analysis, and interpretation of the data, preparation of the manuscript, or the decision to submit this manuscript for publication. We are also grateful to Michael Smith, Matt Forde and Lucy Thompson for their comments on an earlier draft.

\section{Ethical Considerations}

The CATSS has ethical approval from the Regional Ethical Review Board in Stockholm (Dnr 2010/597-31/1). Oral consent has been obtained from all subjects participating in the telephone interview.

\section{Correspondence}

Lisa Dinkler, Gillberg Neuropsychiatry Centre, Institute of Neuroscience and Physiology, University of Gothenburg, Kungsgatan 12, 41119 Gothenburg, Sweden, lisa.dinkler@ gu.se, Tel. +46 (0) 31 3425991, Fax: +46 (0) 31109030 


\section{Key points}

- Child maltreatment has been strongly associated with child psychiatric disorders including neurodevelopmental disorders (NDDs), but the mechanisms behind the association between child maltreatment and NDDs are unclear.

- Maltreated nine-year-old children have a greater number of neurodevelopmental disorders than non-maltreated children.

- Child maltreatment is associated with a small increase in symptoms of at least some neurodevelopmental disorders including ADHD and ASD, yet the direction of causality is unclear. The larger part of the association between child maltreatment and neurodevelopmental disorders is explained by familial factors.

- A full neurodevelopmental assessment is essential for all maltreated children. Parents of children with neurodevelopmental difficulties need additional support. 


\section{References}

Anckarsater, H., Lundstrom, S., Kollberg, L., Kerekes, N., Palm, C., Carlstrom, E., . . Lichtenstein, P. (2011). The Child and Adolescent Twin Study in Sweden (CATSS). Twin Res Hum Genet, 14(6), 495-508.

Arseneault, L., Milne, B. J., Taylor, A., Adams, F., Delgado, K., Caspi, A., \& Moffitt, T. E. (2008). Being bullied as an environmentally mediated contributing factor to children's internalizing problems: a study of twins discordant for victimization. Archives of Pediatrics and Adolescent Medicine, 162(2), 145-150. doi: 10.1001/archpediatrics.2007.53

Berenz, E. C., Amstadter, A. B., Aggen, S. H., Knudsen, G. P., Reichborn-Kjennerud, T., Gardner, C. O., \& Kendler, K. S. (2013). Childhood trauma and personality disorder criterion counts: a co-twin control analysis. Journal of Abnormal Psychology, 122(4), 1070-1076. doi: 10.1037/a0034238

Brown, R. C., Berenz, E. C., Aggen, S. H., Gardner, C. O., Knudsen, G. P., Reichborn-Kjennerud, T., . . . Amstadter, A. B. (2014). Trauma Exposure and Axis I Psychopathology: A Co-twin Control Analysis in Norwegian Young Adults. Psychological Trauma: Theory, Research, Practice and Policy, 6(6), 652-660. doi: 10.1037/a0034326

Capusan, A. J., Kuja-Halkola, R., Bendtsen, P., Viding, E., McCrory, E., Marteinsdottir, I., \& Larsson, H. (2016). Childhood maltreatment and attention deficit hyperactivity disorder symptoms in adults: a large twin study. Psychological Medicine, 1-10. doi: 10.1017/s0033291716001021

D'Onofrio, B. M., Lahey, B. B., Turkheimer, E., \& Lichtenstein, P. (2013). Critical need for family-based, quasi-experimental designs in integrating genetic and social science research. American Journal of Public Health, 103 Suppl 1, S46-55. doi: 10.2105/ajph.2013.301252

Danese, A., Moffitt, T. E., Arseneault, L., Bleiberg, B. A., Dinardo, P. B., Gandelman, S. B., . . Caspi, A. (2016). The Origins of Cognitive Deficits in Victimized Children: Implications for Neuroscientists and Clinicians. American Journal of Psychiatry, O(0), appi.ajp.2016.16030333. doi: 10.1176/appi.ajp.2016.16030333

Felitti, V. J., Anda, R. F., Nordenberg, D., Williamson, D. F., Spitz, A. M., Edwards, V., . . Marks, J. S. (1998). Relationship of childhood abuse and household dysfunction to many of the leading causes of death in adults. The Adverse Childhood Experiences (ACE) Study. American Journal of Preventive Medicine, 14(4), 245-258.

Forsman, M., \& Långström, N. (2012). Child maltreatment and adult violent offending: populationbased twin study addressing the 'cycle of violence' hypothesis. Psychological Medicine, 42(09), 1977-1983. doi: doi:10.1017/S0033291711003060

Gillberg, C. (2010). The ESSENCE in child psychiatry: Early Symptomatic Syndromes Eliciting Neurodevelopmental Clinical Examinations. Research in Developmental Disabilities, 31(6), 1543-1551. doi: 10.1016/j.ridd.2010.06.002

Hart, H., \& Rubia, K. (2012). Neuroimaging of child abuse: a critical review. Frontiers in Human Neuroscience, 6, 52. doi: 10.3389/fnhum.2012.00052

Jaffee, S. R., Caspi, A., Moffitt, T. E., Polo-Tomas, M., Price, T. S., \& Taylor, A. (2004). The limits of child effects: evidence for genetically mediated child effects on corporal punishment but not on physical maltreatment. Developmental Psychology, 40(6), 1047-1058. doi: 10.1037/00121649.40.6.1047

Jaffee, S. R., Caspi, A., Moffitt, T. E., \& Taylor, A. (2004). Physical maltreatment victim to antisocial child: evidence of an environmentally mediated process. Journal of Abnormal Psychology, 113(1), 44-55. doi: http://dx.doi.org/10.1037/0021-843X.113.1.44

Jones, L., Bellis, M. A., Wood, S., Hughes, K., McCoy, E., Eckley, L., . . Officer, A. (2012). Prevalence and risk of violence against children with disabilities: a systematic review and meta-analysis of observational studies. Lancet, 380(9845), 899-907. doi: 10.1016/s0140-6736(12)60692-8

Jonson-Reid, M., Presnall, N., Drake, B., Fox, L., Bierut, L., Reich, W., ... Constantino, J. N. (2010). Effects of child maltreatment and inherited liability on antisocial development: an official records study. Journal of the American Academy of Child and Adolescent Psychiatry, 49(4), 321-332. doi: http://dx.doi.org/10.1016/j.jaac.2009.11.015 
Kendler, K. S., \& Baker, J. H. (2007). Genetic influences on measures of the environment: a systematic review. Psychological Medicine, 37(5), 615-626. doi: 10.1017/s0033291706009524

Kendler, K. S., Bulik, C. M., Silberg, J., Hettema, J. M., Myers, J., \& Prescott, C. A. (2000). Childhood sexual abuse and adult psychiatric and substance use disorders in women: an epidemiological and cotwin control analysis. Archives of General Psychiatry, 57(10), 953-959. doi: 10.1001/archpsyc.57.10.953

Kessler, R. C., McLaughlin, K. A., Green, J. G., Gruber, M. J., Sampson, N. A., Zaslavsky, A. M., . . Williams, D. R. (2010). Childhood adversities and adult psychopathology in the WHO World Mental Health Surveys. British Journal of Psychiatry, 197(5), 378-385. doi: 10.1192/bjp.bp.110.080499

Kocovska, E., Puckering, C., Follan, M., Smillie, M., Gorski, C., Barnes, J., . . Minnis, H. (2012). Neurodevelopmental problems in maltreated children referred with indiscriminate friendliness. Research in Developmental Disabilities, 33(5), 1560-1565. doi: 10.1016/j.ridd.2012.02.016

Kumsta, R., Kreppner, J., Kennedy, M., Knights, N., Rutter, M., \& Sonuga-Barke, E. (2015). Psychological consequences of early global deprivation: An overview of findings from the English \& Romanian Adoptees study. European Psychologist, 20(2), 138-151. doi: http://dx.doi.org/10.1027/1016-9040/a000227

Larson, T., Anckarsater, H., Gillberg, C., Stahlberg, O., Carlstrom, E., Kadesjo, B., . . Gillberg, C. (2010). The Autism - Tics, AD/HD and other Comorbidities inventory (A-TAC): further validation of a telephone interview for epidemiological research. BMC Psychiatry, 10(1), 1.

Larson, T., Lundstrom, S., Nilsson, T., Selinus, E. N., Rastam, M., Lichtenstein, P., . . Kerekes, N. (2013). Predictive properties of the A-TAC inventory when screening for childhood-onset neurodevelopmental problems in a population-based sample. BMC Psychiatry, 13, 233. doi: 10.1186/1471-244X-13-233

Lifford, K. J., Harold, G. T., \& Thapar, A. (2009). Parent-child hostility and child ADHD symptoms: a genetically sensitive and longitudinal analysis. Journal of Child Psychology and Psychiatry and Allied Disciplines, 50(12), 1468-1476. doi: 10.1111/j.1469-7610.2009.02107.x

Luders, E., Narr, K. L., Hamilton, L. S., Phillips, O. R., Thompson, P. M., Valle, J. S., . . Levitt, J. G. (2009). Decreased callosal thickness in attention-deficit/hyperactivity disorder. Biological Psychiatry, 65(1), 84-88. doi: 10.1016/j.biopsych.2008.08.027

McGue, M., Osler, M., \& Christensen, K. (2010). Causal inference and observational research: the utility of twins. Perspectives on Psychological Science, 5(5), 546-556. doi: $10.1177 / 1745691610383511$

Minnis, H. (2013). Maltreatment-associated psychiatric problems: an example of environmentally triggered ESSENCE? ScientificWorldJournal, 2013, 148468. doi: 10.1155/2013/148468

Minnis, H., Reekie, J., Young, D., O'Connor, T., Ronald, A., Gray, A., \& Plomin, R. (2007). Genetic, environmental and gender influences on attachment disorder behaviours. British Journal of Psychiatry, 190, 490-495. doi: 10.1192/bjp.bp.105.019745

Narusyte, J., Neiderhiser, J. M., D’Onofrio, B. M., Reiss, D., Spotts, E. L., Ganiban, J., \& Lichtenstein, P. (2008). Testing different types of genotype-environment correlation: an extended childrenof-twins model. Developmental Psychology, 44(6), 1591-1603. doi: 10.1037/a0013911

Neiderhiser, J. M., Reiss, D., Pedersen, N. L., Lichtenstein, P., Spotts, E. L., Hansson, K., . . Ellhammer, O. (2004). Genetic and environmental influences on mothering of adolescents: a comparison of two samples. Developmental Psychology, 40(3), 335-351. doi: 10.1037/00121649.40.3.335

Neuhaus, J. M., \& McCulloch, C. E. (2006). Separating between- and within-cluster covariate effects by using conditional and partitioning methods. Journal of the Royal Statistical Society: Series B (Statistical Methodology), 68(5), 859-872. doi: 10.1111/j.1467-9868.2006.00570.x

Norman, R. E., Byambaa, M., De, R., Butchart, A., Scott, J., \& Vos, T. (2012). The long-term health consequences of child physical abuse, emotional abuse, and neglect: a systematic review and meta-analysis. PLoS Medicine, 9(11), 1-31. doi: 10.1371/journal.pmed.1001349 
O'Connor, T. G., Deater-Deckard, K., Fulker, D., Rutter, M., \& Plomin, R. (1998). Genotypeenvironment correlations in late childhood and early adolescence: antisocial behavioral problems and coercive parenting. Developmental Psychology, 34(5), 970-981.

Schulz-Heik, R. J., Rhee, S. H., Silvern, L. E., Haberstick, B. C., Hopfer, C., Lessem, J. M., \& Hewitt, J. K. (2010). The association between conduct problems and maltreatment: testing genetic and environmental mediation. Behavior Genetics, 40(3), 338-348. doi: 10.1007/s10519-009-93246

Teicher, M. H., \& Samson, J. A. (2016). Annual Research Review: Enduring neurobiological effects of childhood abuse and neglect. Journal of Child Psychology and Psychiatry and Allied Disciplines, 57(3), 241-266. doi: 10.1111/jcpp.12507

Wolfe, J., \& Kimerling, R. (1997). Gender issues in the assessment of posttraumatic stress disorder. In J. Wilson \& T. M. Keane (Eds.), Assessing Psychological Trauma and PTSD (pp. 192-238). New York: Guilford. 


\section{Concomitant Neurodevelopmental Disorders (NDDs) in twin pairs concordant or discordant for childhood maltreatment}

Figure 1 Each line in the categories MZ and DZ-ss represents a twin pair. Each square under the headings 'Probands' or 'Co-twins' represents an individual. Each square to the right of the proband and to the left of the co-twin squares represents a concomitant NDD (broad definition) of that individual.

\section{Effect of Childhood Maltreatment (CM) on symptoms of Neurodevelopmental Disorders (NDDs)}

Figure 2 Plotted are the z-standardized mean differences between $\mathrm{CM}$ and non-CM children in a) the number of NDDs (broad definition; i.e., the effect of CM exposure on NDD load), and b) the number of symptoms of each NDD separately (i.e., the effect of CM exposure on NDD symptoms). Results for males are displayed in the upper row, results for females in the lower row. The category 'All twins' represents the effect on the population level, i.e. the difference in number of NDDs/NDD symptoms between all CM children vs. all non-CM children, taking no account of twin pair relatedness or zygosity. The categories 'Within DZ-ss pairs' and 'Within MZ pairs' represent the difference in number of NDDs/NDD symptoms between co-twins discordant for CM exposure. Error bars demarcate the confidence intervals; significance was tested at a $5 \%$ level. 


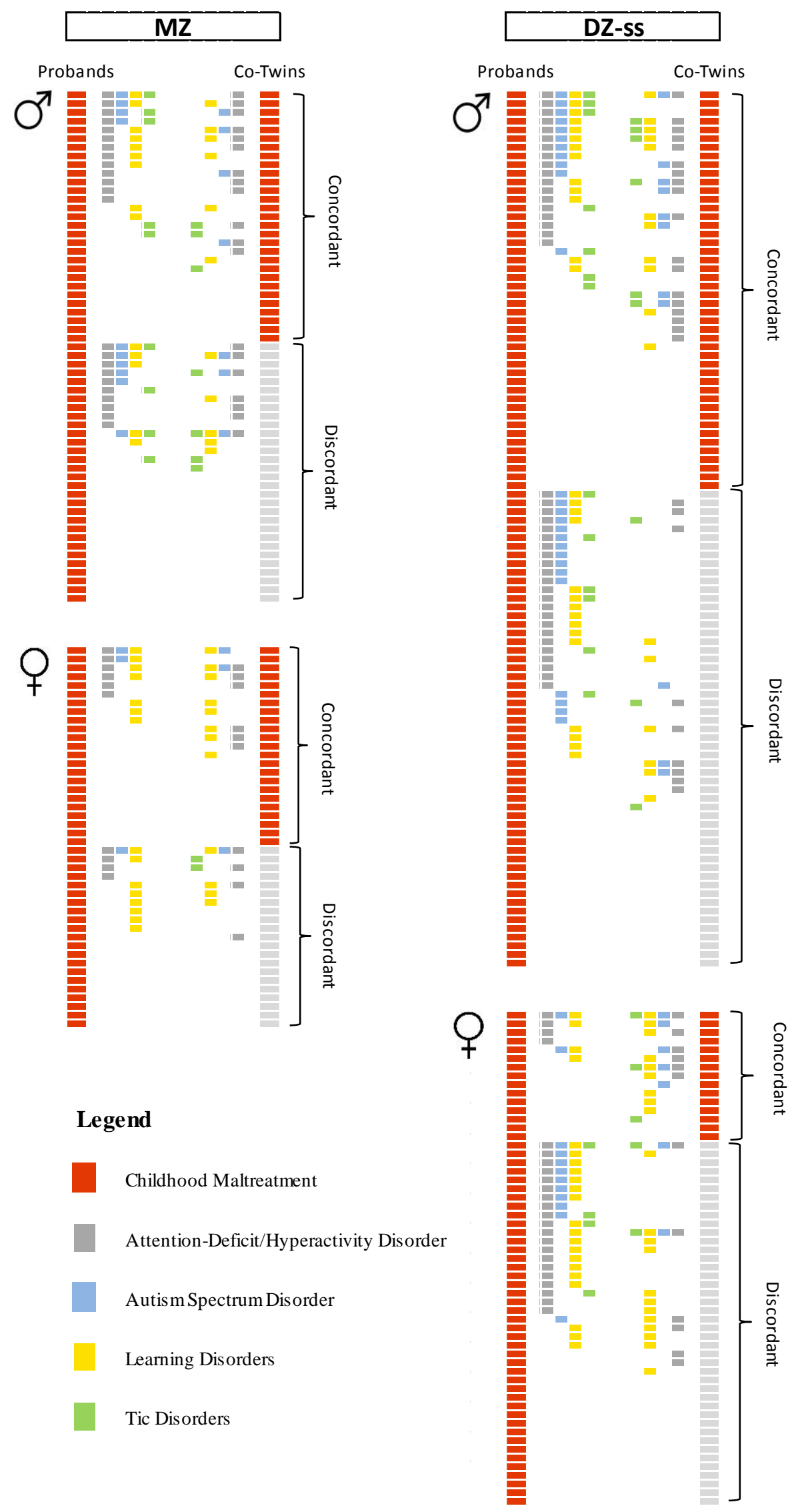



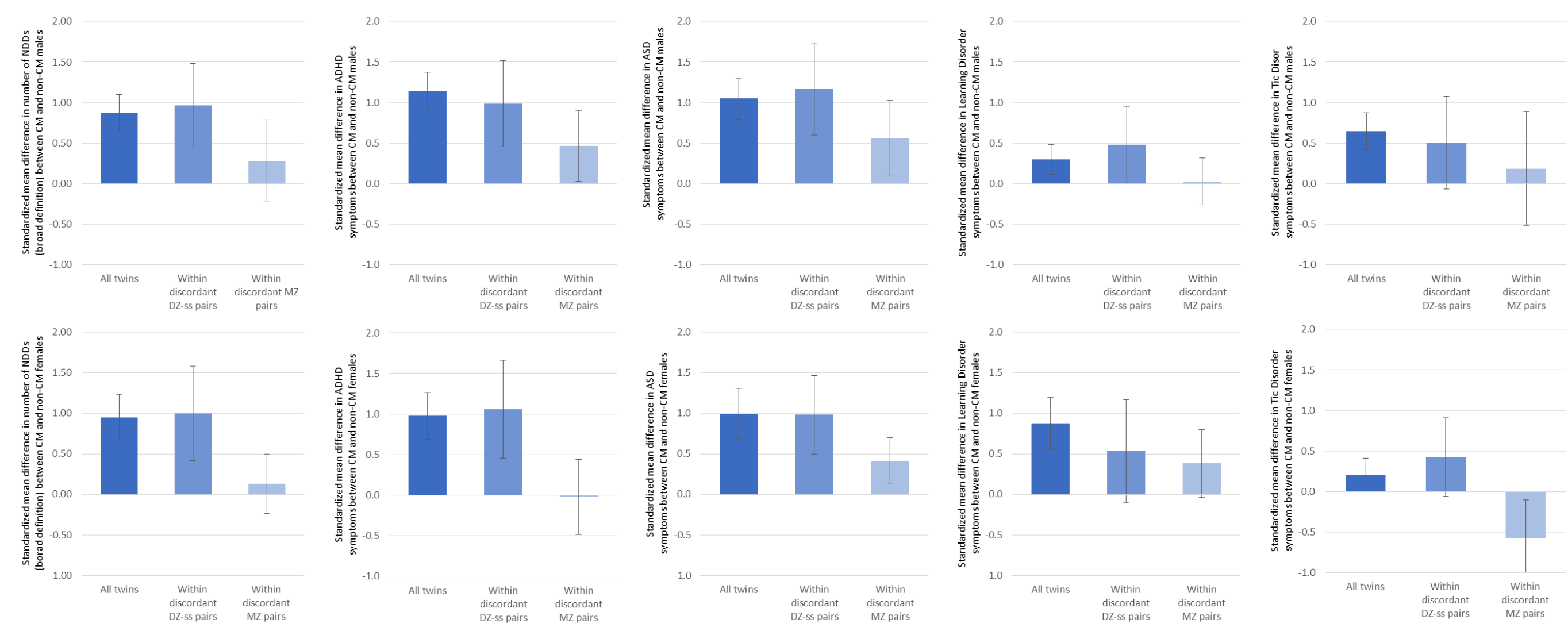
Table 1 Distribution of Neurodevelopmental Problems in 8,192 Swedish twins

\begin{tabular}{|c|c|c|c|c|c|c|c|c|c|}
\hline & & & & & & \multicolumn{4}{|c|}{ By Sex and Child Maltreatment } \\
\hline & \multicolumn{2}{|c|}{ By Sex } & \multicolumn{2}{|c|}{ By Child Maltreatment } & \multicolumn{2}{|r|}{ Male } & \multicolumn{2}{|r|}{ Female } & \multirow[b]{2}{*}{ Total } \\
\hline & Male & Female & $\mathrm{CM}$ & non-CM & $\mathrm{CM}$ & non-CM & $\mathrm{CM}$ & non-CM & \\
\hline $\mathrm{N}$ & 4,143 & 4,041 & 374 & 7,810 & 235 & 3,908 & 139 & 3,902 & 8,184 \\
\hline \multicolumn{10}{|l|}{ Mean Number of NDDs (SD) } \\
\hline $\begin{array}{l}\text { NDDs (broad definition, range } \\
0-4)\end{array}$ & $0.40(0.78)$ & $0.27(0.63)^{* * *}$ & $0.97(1.14)$ & $0.31(0.67)^{* * *}$ & $0.99(1.16)$ & $0.37(0.74) * * *$ & $0.93(1.11)$ & $0.25(0.60)^{* * *}$ & $0.34(0.71)$ \\
\hline $\begin{array}{l}\text { NDDs (narrow definition, range } \\
0-4 \text { ) }\end{array}$ & $0.10(0.40)$ & $0.07(0.32)^{* * *}$ & $0.35(0.72)$ & $0.07(0.33)^{* * *}$ & $0.37(0.70)$ & $0.09(0.37)^{* * *}$ & $0.32(0.74)$ & $0.06(0.29)^{* * *}$ & $0.08(0.36)$ \\
\hline \multicolumn{10}{|l|}{ Mean Number of Symptoms (SD) } \\
\hline ADHD (range 0-19) & $2.49(3.48)$ & $1.75(2.85)^{* * *}$ & $5.45(5.27)$ & $1.97(2.98)^{* * *}$ & $5.88(5.40)$ & $2.29(3.22) * * *$ & $4.73(4.99)$ & $1.64(2.68) * * *$ & $2.13(3.21)$ \\
\hline ASD (range 0-17) & $0.99(1.76)$ & $0.66(1.37)^{* * *}$ & $2.44(2.85)$ & $0.75(1.46)^{* * *}$ & $2.57(2.86)$ & $0.89(1.62) * * *$ & $2.21(2.83)$ & $0.61(1.26)^{* * *}$ & $0.83(1.59)$ \\
\hline Learning Disorders (range 0-3) & $0.31(0.63)$ & $0.26(0.61)^{* * *}$ & $0.60(0.91)$ & $0.27(0.60)^{* * *}$ & $0.48(0.80)$ & $0.30(0.62)^{* * *}$ & $0.79(1.04)$ & $0.24(0.58)^{* * *}$ & $0.29(0.62)$ \\
\hline Tic Disorders (range 0-3) & $0.19(0.50)$ & $0.12(0.39)^{* * *}$ & $0.37(0.71)$ & $0.15(0.43)^{* * *}$ & $0.47(0.78)$ & $0.18(0.48)^{* * *}$ & $0.21(0.54)$ & $0.12(0.38)^{*}$ & $0.16(0.45)$ \\
\hline Prevalence of any NDD (broad & 26.9 & 19.4 & 51.3 & 21.9 & 51.5 & 25.5 & 51.1 & 18.3 & 23.2 \\
\hline definition) in $\%$ [95\% CI] & {$[25.6,28.3]$} & {$[18.2,20.6]$} & {$[46.2,56.4]$} & {$[21.0,22.8]$} & {$[45.1,57.9]$} & {$[24.1,26.8]$} & {$[42.7,59.5]$} & {$[17.1,19.5]$} & {$[22.3,24.1]$} \\
\hline Prevalence of any NDD (narrow & 7.7 & 5.0 & 24.1 & 5.5 & 26.8 & 6.5 & 19.4 & 4.5 & 6.3 \\
\hline definition) in $\%[95 \% \mathrm{CI}]$ & {$[6.8,8.5]$} & {$[4.3,5.6]$} & {$[19.7,28.4]$} & {$[5.0,6.0]$} & {$[21.1,32.5]$} & {$[5.7,7.3]$} & {$[12.8,26.1]$} & {$[3.8,5.1]$} & {$[5.8,6.9]$} \\
\hline
\end{tabular}

Note. $\mathrm{CM}$ /non-CM = exposure/no exposure to childhood maltreatment, $\mathrm{MZ}=$ monozygotic, $\mathrm{DZ}=$ dizygotic same sex, DZ-os = dizygotic opposite sex, NDP =

Neurodevelopmental Problems, NDD = Neurodevelopmental Disorder, ADHD = Attention-Deficit/Hyperactivity Disorder, ASD = Autism Spectrum Disorder.

$* p<.01, * * * p<.001$; comparisons with independent t-Tests (Male vs. Female, CM vs. non-CM) 
Table 2 Sex-specific effects of Childhood Maltreatment (CM) on number and symptoms of Neurodevelopmental Disorders (NDDs) in a Co-Twin Control Design

\begin{tabular}{|c|c|c|c|c|c|c|}
\hline & $N$ & $\begin{array}{c}\text { Number of } \\
\text { discordant } \\
\text { pairs }\end{array}$ & $\begin{array}{l}\text { Unstandardized mean } \\
\text { score on the A-TAC } \\
\text { scale in CM twins }\end{array}$ & $\begin{array}{l}\text { Unstandardized mean } \\
\text { score on the A-TAC } \\
\text { scale in non-CM twins }\end{array}$ & $\begin{array}{c}\text { Standardized } \\
\text { mean difference } \\
\beta\end{array}$ & $p$ \\
\hline \multicolumn{7}{|c|}{ Number of NDDs (broad definition) } \\
\hline \multicolumn{7}{|l|}{ Males } \\
\hline All Twins & 4143 & - & 0.99 & 0.37 & $0.87(0.64-1.10)$ & $<.0001$ \\
\hline Within DZ-ss pairs & 2391 & 55 & 1.07 & 0.38 & $0.97(0.45-1.48)$ & .0002 \\
\hline Within MZ pairs & 1752 & 30 & 0.83 & 0.63 & $0.28(-0.23-0.79)$ & .2800 \\
\hline \multicolumn{7}{|l|}{ Females } \\
\hline All Twins & 4041 & - & 0.93 & 0.25 & $0.95(0.67-1.23)$ & $<.0001$ \\
\hline Within DZ-ss pairs & 2219 & 42 & 1.24 & 0.52 & $1.00(0.42-1.58)$ & .0007 \\
\hline Within MZ pairs & 1822 & 21 & 0.62 & 0.52 & $0.13(-0.23-0.50)$ & .4740 \\
\hline \multicolumn{7}{|l|}{ ADHD symptoms } \\
\hline \multicolumn{7}{|l|}{ Males } \\
\hline All Twins & 4126 & - & 5.88 & 2.29 & $1.13(0.89-1.38)$ & $<.0001$ \\
\hline Within DZ-ss pairs & 2380 & 55 & 6.02 & 2.90 & $0.99(0.45-1.52)$ & .0003 \\
\hline Within MZ pairs & 1746 & 30 & 6.12 & 4.65 & $0.46(0.03-0.90)$ & .0372 \\
\hline \multicolumn{7}{|l|}{ Females } \\
\hline All Twins & 4033 & - & 4.73 & 1.64 & $0.98(0.69-1.27)$ & $<.0001$ \\
\hline Within DZ-ss pairs & 2214 & 42 & 6.50 & 3.14 & $1.06(0.46-1.67)$ & .0006 \\
\hline Within MZ pairs & 1819 & 21 & 3.26 & 3.33 & $-0.02(-0.48-0.44)$ & .9236 \\
\hline \multicolumn{7}{|l|}{ ASD symptoms } \\
\hline \multicolumn{7}{|l|}{ Males } \\
\hline All Twins & 4136 & - & 2.57 & 0.89 & $1.05(0.79-1.30)$ & $<.0001$ \\
\hline Within DZ-ss pairs & 2386 & 55 & 3.09 & 1.22 & $1.17(0.60-1.73)$ & $<.0001$ \\
\hline Within MZ pairs & 1750 & 30 & 2.65 & 1.75 & $0.56(0.09-1.03)$ & .0185 \\
\hline \multicolumn{7}{|l|}{ Females } \\
\hline All Twins & 4036 & - & 2.21 & 0.61 & $1.00(0.69-1.31)$ & $<.0001$ \\
\hline Within DZ-ss pairs & 2215 & 42 & 2.82 & 1.24 & $0.99(0.50-1.47)$ & $<.0001$ \\
\hline Within MZ pairs & 1821 & 21 & 1.69 & 1.02 & $0.41(0.13-0.70)$ & .0044 \\
\hline
\end{tabular}


Table 2 Sex-specific effects of Childhood Maltreatment (CM) on number and symptoms of Neurodevelopmental Disorders (NDDs) in a Co-Twin Control Design (continued)

\begin{tabular}{|c|c|c|c|c|c|c|}
\hline & $N$ & $\begin{array}{c}\text { Number of } \\
\text { discordant } \\
\text { pairs } \\
\end{array}$ & $\begin{array}{c}\text { Unstandardized mean } \\
\text { score on the A-TAC } \\
\text { scale in CM twins }\end{array}$ & $\begin{array}{l}\text { Unstandardized mean } \\
\text { score on the A-TAC } \\
\text { scale in non-CM twins }\end{array}$ & $\begin{array}{c}\text { Standardized } \\
\text { mean difference } \\
\beta\end{array}$ & $p$ \\
\hline \multicolumn{7}{|c|}{ Learning Disorder symptoms } \\
\hline \multicolumn{7}{|l|}{ Males } \\
\hline All Twins & 4143 & - & 0.48 & 0.30 & $0.30(0.11-0.48)$ & .0014 \\
\hline Within DZ-ss pairs & 2391 & 55 & 0.54 & 0.24 & $0.48(0.02-0.94)$ & .0405 \\
\hline Within MZ pairs & 1752 & 30 & 0.32 & 0.30 & $0.03(-0.27-0.32)$ & .8575 \\
\hline \multicolumn{7}{|l|}{ Females } \\
\hline All Twins & 4041 & - & 0.79 & 0.24 & $0.88(0.57-1.20)$ & $<.0001$ \\
\hline Within DZ-ss pairs & 2219 & 42 & 0.90 & 0.57 & $0.54(-0.10-1.17)$ & .0999 \\
\hline Within MZ pairs & 1822 & 21 & 0.57 & 0.33 & $0.38(-0.04-0.80)$ & .0737 \\
\hline \multicolumn{7}{|l|}{ Tic Disorder symptoms } \\
\hline \multicolumn{7}{|l|}{ Males } \\
\hline All Twins & 4140 & - & 0.47 & 0.18 & $0.64(0.41-0.87)$ & $<.0001$ \\
\hline Within DZ-ss pairs & 2388 & 55 & 0.48 & 0.25 & $0.50(-0.07-1.08)$ & .0844 \\
\hline Within MZ pairs & 1752 & 30 & 0.42 & 0.33 & $0.18(-0.52-0.89)$ & .6066 \\
\hline \multicolumn{7}{|l|}{ Females } \\
\hline All Twins & 4037 & - & 0.21 & 0.12 & $0.20(0.00-0.41)$ & .0541 \\
\hline Within DZ-ss pairs & 2216 & 42 & 0.33 & 0.14 & $0.42(-0.06-0.91)$ & .0881 \\
\hline Within MZ pairs & 1821 & 21 & 0.02 & 0.29 & $-0.58(-1.06--0.10)$ & .0171 \\
\hline
\end{tabular}

Note. The standardized regression coefficient $\beta$ corresponds to the mean difference in the number of NDDs/number of symptoms of specific NDDs between CM and non-CM children. The category 'All twins' represents effect on the population level, i.e. the mean difference between all CM children vs. all non-CM children, taking no account of twin pair relatedness or zygosity. The categories 'Within DZ-ss pairs' and 'Within MZ pairs' represents the mean difference between co-twins discordant for CM exposure. 